\title{
THE CHEMICAL EVOLUTION OF THE MAGELLANIC CLOUDS
}

\author{
S.C. RUSSELL \\ Dublin Institute for Advanced Studies \\ 5 Merrion Square \\ Dublin 2 \\ Ireland
}

\begin{abstract}
The chemical and structural evolution of the Magellanic Clouds are compared with those of the Galaxy. Simple models with gas infall and bimodal star formation are assumed, and are constrained by the most recent observational data. The evolution of the gas content, and the $\mathrm{He}, \mathrm{C}, \mathrm{O}$ and $\mathrm{Fe}$ abundances are all successfully modelled. The abundance of $\mathrm{N}$ is, however, predicted too high, and the apparent ages of the oldest star clusters are inconsistent with the simple model.
\end{abstract}

\section{Introduction}

The first fully consistent survey of the detailed abundances in the Magellanic Clouds was carried out by Russell and Bessell (1989) and Russell and Dopita (1990a). The survey depended on analysis of spectra from a selection of F-supergiants, HII regions and supernova remnants in each of the Clouds. A full discussion of the results and subsequent modelling is given in Russell and Dopita (1990b), so we will confine ourselves here to a brief review of our modelling of the data.

\section{The Model}

The modelling code adopted was developed along the lines described by Matteucci and Tornambe (1985) and Matteucci and Greggio (1986). The gas is assumed to be infalling with an exponential time constant of $\tau_{\text {inf }}$, and star formation is assumed to be bimodal in character as proposed by Güsten and Mezger (1982). At each time step the amount of infallen gas, the numbers of stars that have born and died in each mass bin, and the consequent changes in gas composition are calculated.

The critical parameters in the modelling process are: (a) the power law exponent $p$ of the high mass $\left(>1 \mathrm{M}_{\odot}\right)$ initial mass function (IMF); (b) the gas infall timescale $\tau_{\text {inf }}$ (c) the gas depletion timescale $\tau_{\text {depl }}$, defined as the instantaneous gas mass divided by the high mass star formation rate (SFR); (d) the deflagration timescale $\tau_{\text {defl }}$, defined as the characteristic time between the formation of a white dwarf binary pair, and the subsequent deflagration of one of them as a Type Ia supernova; (e) the percentage of white dwarfs that form binaries; (f) the ratio of low mass to high mass SFRs. The slope of the IMF is fixed mainly by the observed oxygen abundance, while the percentage of binaries and $\tau_{\text {defl }}$ together are fixed by the iron abundance. The gas infall timescale is determined by the observed time of maximum star formation, and the ratio of gas mass to total mass fixes the gas depletion timescale. The ratio of low- to high-mass SFRs was chosen to be the same as that derived by Dopita (1989) for the Galaxy. Modelling showed that even a $25 \%$ error in 
this parameter would only result in a 5\% error in the IMF slope. The major effect would be an increased uncertainty in the percentage of binaries or the deflagration timescale $\tau_{\text {defl }}$. These latter two parameters are poorly determined anyway, and signify only that the iron abundance provides poor constraints on a chemical evolution model.

\section{Discussion}

The results of our modelling are summarized in Table 1, where the fitted parameters are given in bold type. The IMF exponents agree well with the observed values from Humphreys and McElroy (1984), even though they were not fitted parameters. The most consistent models were obtained assuming an evolution time of 8 Gyrs. This is a somewhat grey area since on the one hand, star clusters have been found in both Clouds that have ages of 10 gyrs or more (see e.g. Bica et al. 1986, Frantsman 1988). On the other hand, the models might be telling us that except in isolated clumps, the global collapse of the gas clouds may only have begun in earnest about 8 Gyrs ago.

The peak of star formation was chosen to lie well within the range of possible values. Further modelling, however, showed that if the youngest possible peaks in the SFRs suggested in the literature were assumed, other parameters would be stretched beyond credibility. The final three physical parameters in Table 1 are poorly constrained by observations, but are probably correct to better than a factor of two.

Helium, and more importantly, carbon are well predicted by the models. The observed carbon abundances are derived from the spectra from F-supergiants (Russell and Bessell 1989), and are in conflict with the much lower abundances derived by Dufour et al. (1982) from the UV spectra of HII regions. Our models support the higher carbon abundances measured in the supergiants, and indicate that it is the HII region abundances that are anomalous.

Finally, the models overestimate the nitrogen abundances in the Clouds as determined from observations of HII regions. Supernova remnants observed by Russell and Dopita (1990a) and the B-stars studied by Reitermann et al. (1990) indicate somewhat higher nitrogen abundances. It may be that HII regions are again returning anomalous results.

Table 1. Physical Parameters

\begin{tabular}{|c|c|c|c|c|c|}
\hline \multirow{2}{*}{ Paramcler } & \multicolumn{2}{|c|}{ SMC } & \multicolumn{2}{|c|}{ LMC } & \multirow{2}{*}{ Reference } \\
\hline & Litcrature $N$ & Model & Litcraturc 1 & Model & \\
\hline IMF exponent $p$ & 2.14 & 2.32 & 2.29 & 2.20 & (1) \\
\hline Ratio of gas mass to total mass & 0.36 & 0.36 & 0.11 & 0.11 & (2) \\
\hline Evolution time (Gyrs) & 10.0 & 8.0 & 10.0 & 8.0 & (3) \\
\hline Gas depletion timescalc (Gyrs) & $1.7-6.0$ & 2.9 & $0.6-1.5$ & 1.85 & (4) \\
\hline Gas infall timcscalc (Gyrs) & & 3.0 & & 3.0 & model \\
\hline Peak star formation (Gyrs from present) & t) $2.0-4.0$ & 3.6 & 4.0-7.0 & 5.1 & (4) \\
\hline Low- to high-mass SFR ratio & $\ldots$ & 4.0 & $\ldots$ & 4.0 & model \\
\hline Lifclime of binaries ( $\tau(\mathrm{def})$ ) (Gyrs) & $\ldots$ & 1.5 & $\ldots$ & 1.1 & model \\
\hline$\%$ of stars forming binarics & $\ldots$ & 2.5 & $\ldots$ & 2.0 & model \\
\hline $\mathrm{N}(\mathrm{Hc} / \mathrm{H})$ & 0.081 & 0.082 & 0.089 & 0.091 & (5) \\
\hline [C/II] & -0.86 & $-(0.83$ & -0.58 & -0.32 & (6) \\
\hline $\mathrm{N} / \mathrm{H}$ & -1.31 & -0.62 & -0.80 & -0.24 & (5) \\
\hline$[\mathbf{O} / \mathbf{H}$ & -0.77 & -0.76 & -0.44 & -0.44 & (5) \\
\hline [Fe//H] & -0.65 & -0.64 & $-\mathbf{0 . 3 0}$ & $-\mathbf{0 . 3 0}$ & (6) \\
\hline
\end{tabular}

References: (1) = Humphreys and McElroy (1984), (2) = Lequeux (1984), (3) = Frantsman (1988), (4) = Dopita (1987) and references therein, (5) = Russell and Dopita (1990a), (6) = Russell and Bessell (1989). 


\section{References}

Bica, E., Dottor, H. and Pastoriza, M. (1986), Astr. Ap. 156, 261.

Dopita, M.A. (1987), IAU Symposium 115, Star Forming Regions, M Peimbert and J. Jugaku (eds.), (Reidel: Dordrecht), p. 501.

Dopita, M.A. (1989), The star-gas cycle in galaxies (preprint).

Dufour, R.J., Shields, G.A. and Talbot, R.J. Jr (1982), Ap. J. 252, 461.

Frantsman, Ju. L. (1988), Ap. Spac. Sci. 145, 287.

Güsten, R. and Mezger, P.G. (1982), Vistas in Astr. 26, 159.

Humphreys, R.M. and McElroy, D.G. (1984), Ap. J. 284, 565.

Lequeux, J. (1984), IAU Symposium 108, Structure and Evolution of the Magellanic Clouds, S. van den Bergh and K. S. de Boer (eds), (Reidel: Dordrecht), p. 67.

Matteucci, F. and Greggio, L. (1986), Astr. Ap. 154, 279.

Matteucci, F. and Tornambe, A. (1985), Astr. Ap. 142, 13.

Reitermann, A., Baschek, B., Stahl, O. and Wolf, B. (1990), Astr. Ap. (in press).

Russell, S.C. and Bessell, M.S. (1989), Ap. J. Suppl. 70, 865.

Russell, S.C. and Dopita, M.A. (1990a), Ap. J. Suppl. (in press).

Russell, S.C. and Dopita, M.A. (1990b), Ap. J. (submitted). 